\title{
CECE: A Deep Throttling Demonstrator Cryogenic Engine for NASA's Lunar Lander
}

\author{
Victor J. Giuliano ${ }^{1}$, Timothy G. Leonard ${ }^{2}$, and Walter M. Adamski ${ }^{3}$ \\ Pratt \& Whitney Rocketdyne, West Palm Beach, FL 33410 \\ and \\ Tony S. $\mathrm{Kim}^{4}$ \\ NASA Marshall Space Flight Center, Huntsville, AL 35812
}

\begin{abstract}
As one of the first technology development programs awarded under NASA's Vision for Space Exploration, the Pratt \& Whitney Rocketdyne (PWR) Deep Throttling, Common Extensible Cryogenic Engine (CECE) program was selected by NASA in November 2004 to begin technology development and demonstration toward a deep throttling, cryogenic Lunar Lander engine for use across multiple human and robotic lunar exploration mission segments with extensibility to Mars. The CECE program leverages the maturity and previous investment of a flight-proven hydrogen/oxygen expander cycle engine, the RL10, to develop and demonstrate an unprecedented combination of reliability, safety, durability, throttlability, and restart capabilities in a high-energy, cryogenic engine. NASA Marshall Space Flight Center and NASA Glenn Research Center personnel were integral design and analysis team members throughout the requirements assessment, propellant studies and the deep throttling demonstrator elements of the program. The testbed selected for the initial deep throttling demonstration phase of this program was a minimally modified RL10 engine, allowing for maximum current production engine commonality and extensibility with minimum program cost. In just nine months from technical program start, CECE Demonstrator No. 1 engine testing in April/May 2006 at PWR's E06 test stand successfully demonstrated in excess of 10:1 throttling of the hydrogen/oxygen expander cycle engine. This test provided an early demonstration of a viable, enabling cryogenic propulsion concept with invaluable system-level technology data acquisition toward design and development risk mitigation for both the subsequent CECE Demonstrator No. 2 program and to the future Lunar Lander Design, Development, Test and Evaluation effort.
\end{abstract}

This paper describes the characteristics of the program and the engine design features, as well as component manufacturing efforts, test facility modifications and test results.

\footnotetext{
${ }^{1}$ Program Manager, Constellation Programs, P.O. Box 109600, M/S 710-99, Senior Member

${ }^{2}$ Chief Engineer, Constellation Programs, P.O. Box 109600, M/S 710-99

${ }^{3}$ Manager, Propulsion Systems Analysis and Integration, P.O. Box 109600, M/S 712-67

${ }^{4}$ Lox Hydrogen Deep Throttling Engine (DTE) Advanced Capability Development Project Manager, Science \& Mission Systems Office, Exploration Advanced Capabilities Development Office, NASA/MSFC/VP33, Member
} 


\section{Introduction}

The main goal of the CECE program is to leverage the maturity and previous investment of a flight-proven expander cycle engine to develop and demonstrate technology advancement toward an unprecedented combination of extended reliability, safety, throttlability, and restart capabilities in a high-energy, cryogenic engine.

The resulting technologies may then enable a deep throttling, highly reliable engine for use across most human and robotic exploration mission phases, including: earth-to-orbit, trans-lunar insertion (TLI), lunar orbit insertion (LOI), planetary descent/ascent, trans-Earth insertion (TEI), and Earth orbit insertion (EOI). In formulating the program, consideration was also given to the requirements and program impacts of potential alternate in-situ generated propellants (methane [CH4] and carbon monoxide [CO]). This vision of a common, extensible cryogenic engine, as shown in Figure 1, seeks to enhance Exploration affordability and sustainability objectives through commonality and modularity across lunar mission segments and Mars architecture extensibility by potentially eliminating the need for several "point design" engines.

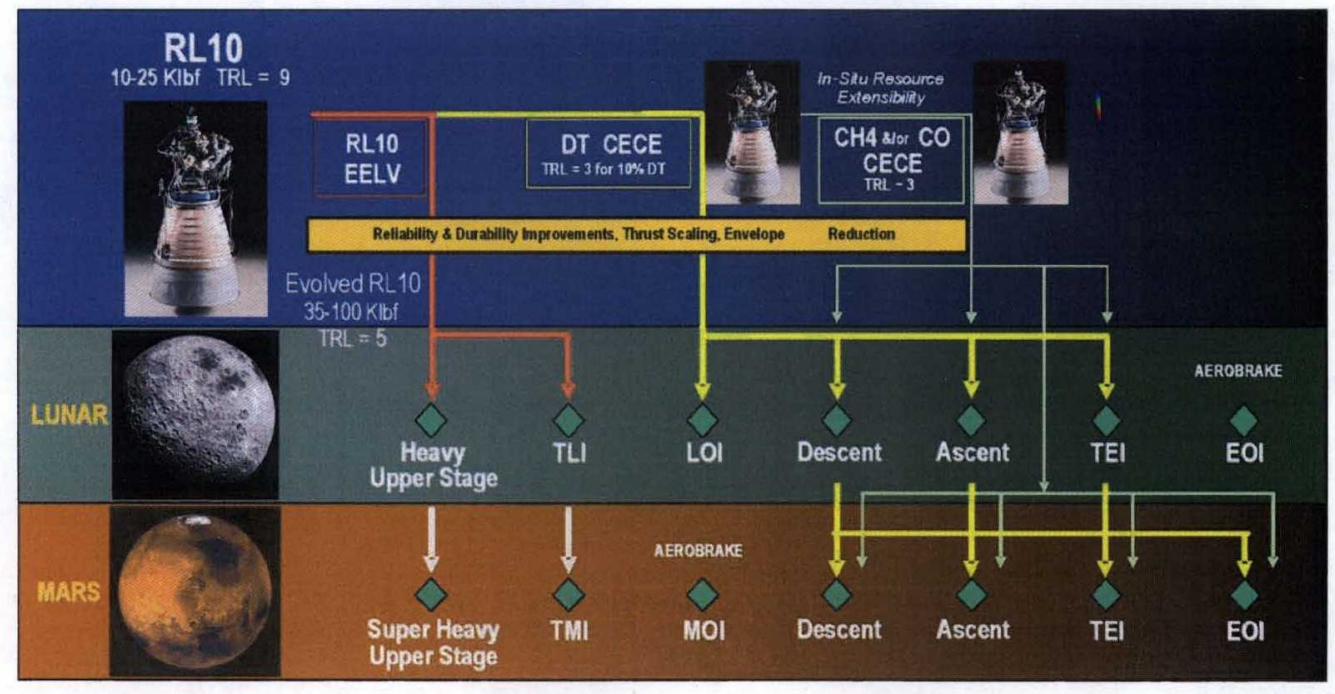

Figure 1: CECE Roadmap for Common, Extensible Propulsion Supporting VSE Mission Segments

For this program, PWR had proposed leveraging the high-performance expander cycle engine for Exploration technology insertion because it offers cycle-unique benefits: robust ignition/restart capability, benign operating conditions, and safe shutdown capability. The RL10 itself benefits from 45 years of production and a high degree of demonstrated reliability. Its specific impulse (Isp) of $465 \mathrm{sec}$ (for the RL10B-2) is the highest of any liquid propellant engine in production. RL10 development efforts oriented toward in-space lunar exploration missions have included durability, combustion stability, contamination, high vacuum, micro-meteoroid damage, multiple start capability, propellant system conditioning, mixture ratio, throttling, hypergolic and redundant ignition, low idle thrust, and engine system operability using methane and propane as alternate propellants (references 1-4). In the 1960s, PWR conducted proof-of-concept deep throttling testing with an early model (RL10A-3) of the RL10 engine. Although the engine was able to operate at a throttle setting of 11 percent thrust, the detailed data necessary to understand engine environments and consequent engine margins for reliability, safety, restarts, re-use etc., was not part of the effort.

The Phase I Program was designed to provide an early demonstration of the CECE's throttling capability and to provide enabling component and system-level data acquisition environments for the enhanced capability design to be developed and demonstrated in Phase II. Phase I was composed of three primary technical tasks: 1) mission requirements and technical performance measures (TPMs) definition consistent with evolving Lunar Lander propulsion system requirements; 2) full-engine system 10:1 deep throttling demonstration and acquisition of risk 
reduction-enabling performance data through the Demo 1 engine test; and 3) conceptual design of a CECE Demonstrator No. 2 configuration toward further risk reduction design and demonstration efforts under Phase II, including preliminary evaluation of methane and carbon-monoxide as alternate propellants. At the conclusion of the program, an engine based on technologies developed and demonstrated during the CECE program would be ready to enter Design, Development, Test and Evaluation (DDT\&E).

The remainder of this paper addresses activities and results achieved under the second technical task (CECE Deep Throttling Demonstrator No. 1 Engine Test), including component and engine design features and manufacturing efforts, test facility modifications and overall results.

\section{CECE Engine Configuration}

The CECE Demonstrator No.1 Engine was assembled from a mix of heritage RL10 development hardware, valves from different programs, and specially made parts needed to meet configuration and performance needs. All of the heritage RL10 development hardware, all of the valves, and all of the assembly manpower to assemble the engine were provided by PWR at no charge to the contract.

Demo 1 was designed to provide the critical empirical engine data necessary to address future operation issues associated with up to a 20:1 throttling range. System-level issues directly impacting the Demo 1 system hardware design included: sufficient control authority to enable system operation to 10 percent thrust, system stability during all throttle points, adequate coolant flow at all conditions, robust ignition, thrust chamber heat flux at all conditions, and the minimum allowable LOX-side injector pressure drop during low throttle points. Hardware changes addressing these issues consisted of a valve suite selection that added the necessary system control flexibility and modifications to critical injector features. Descriptions of these hardware changes are presented over the next several paragraphs.

\section{A. Valves}

The valve suite selected for the CECE Demonstrator No. 1 engine is shown in Figure 2. These valves control chamber pressure $(\mathrm{Pc})$ controlling the turbopump speed, while also maintaining control over mixture ratio (MR). Sufficient turbine bypass was achieved by adding an electromechanical actuator (EMA) control to the RL10 thrust control valve (TCV), as was done on the RL10A-5 (3:1 throttling engines used in the 1990s' DC-X reusable booster stage flight program) and by supplementing the existing bypass flow route with an EMA-controlled RL60 fuel turbine bypass valve (TBV). These complementary flowpaths work in conjunction to determine the proper amount of fuel flow passing through or bypassing the turbine, thus controlling turbopump power output, and

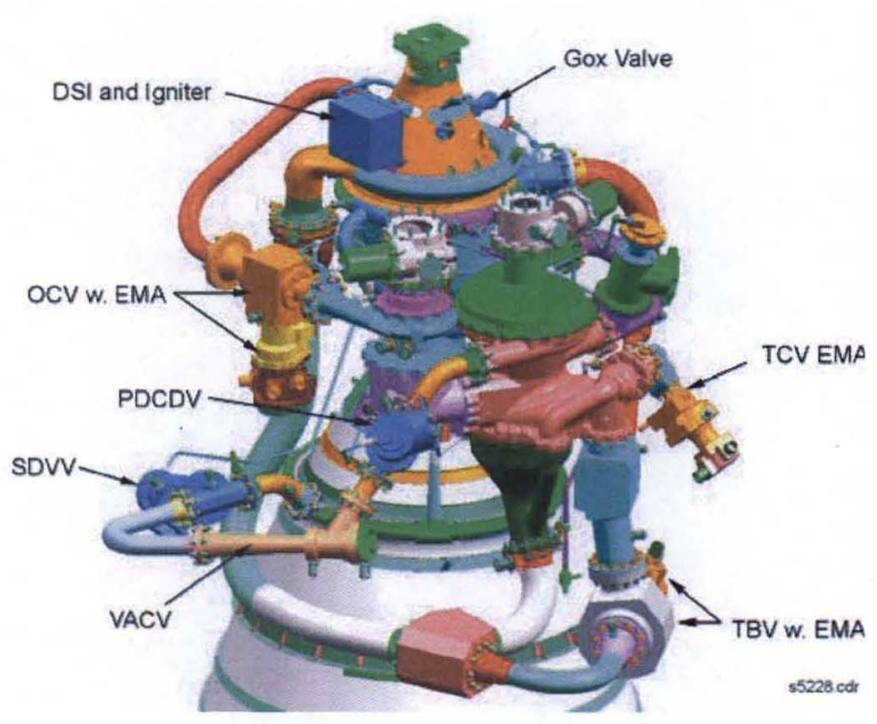

Figure 2: CECE Unique Valve Configuration subsequent chamber pressure and thrust level. The addition of an externally mounted TBV required that fuel flow from the thrust chamber exit manifold be split between the turbine and the new bypass route and be rejoined downstream for final routing to the main fuel shutoff valve (MFSOV) and entry into the injector. Splitting the fuel flow upstream of the turbine is the flow splitter mounted directly between the turbine housing inlet flange, and the TBV inlet flange. Flow entering the flow splitter travels either into the turbine or into the TBV. Downstream of the 
turbine and TBV, the flowpaths rejoin at the flow uniter before continuing on to the MFSOV. The proposed manifold route was also approved following a system structural analysis.

Ten separate large line external manifold assemblies and detail flowpath parts were designed specifically for use on Demo 1. Each manifold was configured as closely as possible to analogous RL10 hardware, and were produced by the RL10 production external tube supplier. In other words, materials, flange configurations, bolt patterns, manufacturing processes, proof pressure testing, and quality inspections were maintained from analogous hardware, if such hardware existed. Where no analogous hardware existed, RL10 production hardware margins and Standard Work methods were employed to assure that the CECE designs met, or exceeded, RL10 production margins. In some cases, the final configuration differed from the original design intent to take advantage of salvageable material on hand, to address manufacturing errors, or due to unavailability of some design-intent seals.

\section{B. Injector/Chamber}

The RL10 injector was redesigned for CECE while maintaining as much commonality with the current RL10 basic injector assembly as possible. The CECE cycle data were used to modify the injector with the principle changes being a reduction of the liquid oxygen flow area. In addition to changing the injector element flow areas, the outer row mixture ratio was reduced. This provided added thermal margin for the chamber and guarded against test program uncertainties.

The CECE cycle was designed to demonstrate 10:1 throttling across a fixed geometry injector. However, since a 10:1 throttle ratio implies a high LOX pressure drop ratio, high pressure drops at 100 percent and low pressure drops at 10 percent were tolerated in the design.

Since the fuel effective flow area was similar to that of the base RL10 engine, the fuel areas needed little modification. The main modification to the injector flow area was to the LOX flow area.

The chamber pressures and higher than heritage LOX injector pressure drops for the cycle operation of CECE required a structural analysis to be conducted on the injector to ensure operation within safe margins. The structural analysis showed stresses were less than those experienced by the injectors on previous RL10 engines and provided sufficient margin.

The final task addressing injector operation was a heat transfer analysis of the CECE injector face transpiration cooling. The faceplate material used for the CECE injector is the same used on the RL10A-4 and RL10B-2 engines. Therefore, the adequacy of the transpiration cooling was assessed for the CECE operating conditions and was found to be adequate.

The CECE combustion chamber was based on a Bill-of-Material A-4 chamber. The thermal loading of the CECE chamber was evaluated at all power levels to ensure chamber health throughout the range of operation. Chamber wall temperature profiles were evaluated at 100 percent, 80 percent, 60 percent, 40 percent, 20 percent; and 10 percent power conditions. It was determined that the wall temperatures would remain lower than those on the RL10A-4 engines throughout the entire range of operation. However, as previously discussed; the outer row mixture ratio was reduced and this added to the predicted chamber thermal margins.

The coolant distribution inside of the CECE dual exit coolant manifold was modeled to assess the uniformity of the coolant and to determine the minimum tube coolant flow rate used in the aforementioned tube hot-wall temperature analysis. These data were used to calculate the hot-wall temperature at off-nominal conditions to assess the maximum thermal loading on the combustion chamber wall. The analysis indicated that the minimum tube coolant flow rate for the cycle occurs at the 100 percent throttle point and is 97 percent of the average tube coolant flow rate. Due to the decrease in pressure loss in the coolant exit manifold as the engine is throttled down, the coolant distribution becomes more evenly distributed as the thrust is decreased. Therefore, the 100 percent condition produces the most extreme coolant non-uniform distribution during operation. It was determined that the coolant distribution provided by the chamber was sufficient to avoid excessive thermal loads within the combustion chamber wall under steady-state operation at all operating conditions. 


\section{Turbomachinery}

The turbopump configuration selected for CECE Demonstrator No. 1, while unique from previous development test versions, was essentially common to the current RL10 production pump and can generally be described as a downchange RL10A-4 configuration. The CECE Demonstrator No. 1 Turbopump Assembly configuration is shown in Figure 3.

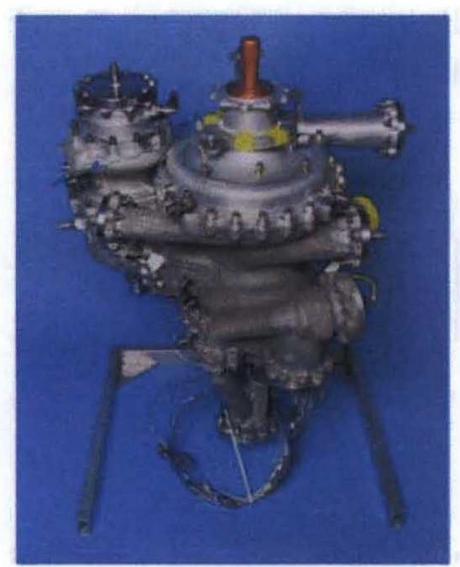

Figure 3. CECE Demonstrator No.1 Pump

The RL10 engine uses a geared turbopump arrangement that allows both fuel and oxidizers pumps to be driven by a common turbine. This ensures both pumps are synchronized in operation, and also allows a simplified control system, with only one valve required to control turbine power input (and correspondingly, engine thrust).

The pumps were assembled from both new and used hardware with the majority of the used components being taken from a previous development turbopump assembly. During the assembly, the turbopumps were refurbished in accordance with the RL10 Engine Turbopump Zero Time Refurbishment procedure, which drives replacement of used gears, bearings, seals and other critical or non-reusable components with new hardware. Each of the gears was processed in-house to the latest coating requirements. Ball bearings for the fuel and LOX pump were specifically selected to ensure that each was suited for CECE operation. This selection was made based upon initial thrust balance modeling and a review of secondary bearing coolant flows.

\section{E06 Test Facility Modifications}

Testing was conducted on PWR's E06 test facility (Figure 4). The E06 test facility, built in 1962, is currently used for development and production testing of RL10 and related rocket engines. While largely well suited to support the planned testing, limited facility vacuum, software, and hardware modifications were needed to control and operate the engine at deep throttle points.

\section{A. Steam System Modifications}

Due to the low throttle conditions expected during CECE demonstration testing, improvements to the facility's pump-out capability were deemed necessary. By connecting a secondary steam ejector system in parallel with the main engine exhaust ejector, additional margin in maintaining the diffuser in a "started" condition at low engine thrusts could be realized.

A secondary ejector system was connected to the heat exchanger directly above the tie in point for the primary ejector system. A spectacle flange located in the additional plumbing immediately upstream of the heat exchanger tie in point was also installed. This spectacle flange can be rotated between engine tests to either allow the secondary ejector system to add additional pump-out capability to the facility, or isolate the secondary system from the primary.

A steam depletion test demonstrated that, with the dual stage ejector systems in parallel, sufficiently low capsule pressures could be maintained for approximately $500 \mathrm{sec}$ without engine flow. Post-CECE Demo 1 testing data allowed a performance comparison between pre-test performance

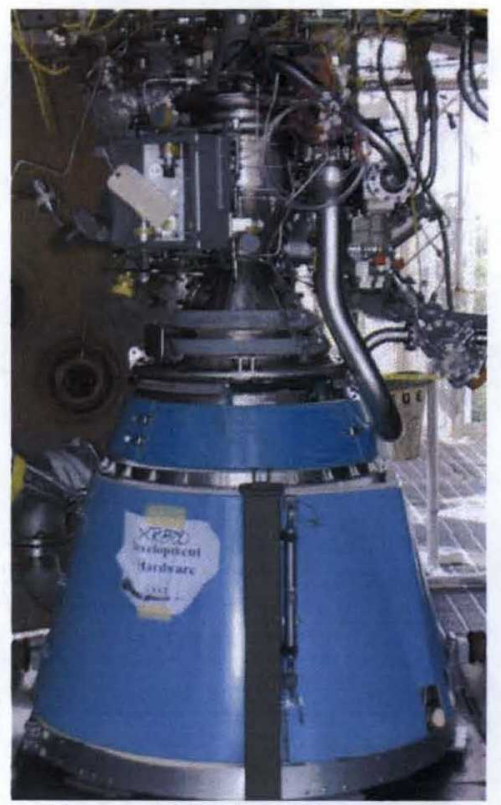

Figure 4: Engine Mounted in E06 Facility estimates and those demonstrated during Demo 1 testing. 


\section{B. Control System Modifications}

In addition to the mechanical modifications needed to run CECE on the E06 test stand, many control and electrical modifications were needed as well. Most significantly, the testing required an integrated control approach that melded several unique control elements into a unified system. Since the program was constructed purely as a demonstrator concept, the systems used to support the program were selected from the best of those available. The control valve suite selected necessitated combining hydraulic, analog, and digital control elements. Additionally, an active control computer had to be integrated into the test facility's existing systems which lacked the capability to perform the control functions desired but nevertheless needed to be retained to operate the facility and engine functions common to RL10.

This approach not only effectively united the many diverse elements used to control CECE, but allowed complete integration with the off-line engine model and run program inputs to enable a full, active simulation prior to the execution of each test.

\section{Engine Test and Results Summary}

Overall, the CECE Demonstrator No. 1 engine completed a total of eight firings for a total of $936.3 \mathrm{sec}$ of operation during this phase of the program. Throughout the program, the engine demonstrated successful ignition and operation across a wide range of operating conditions. By the completion of testing, the engine had operated from 102 percent power down to 9 percent power, all with minimal alterations to the base RL10 system.

The first hot-fire test was performed on 13 April 2006 with a run duration of $2.7 \mathrm{sec}$. The engine had ignition on the first spark (Figure 5) but advanced due to the engine accelerating slower than expected. An error in the valve schedule was found and corrected.

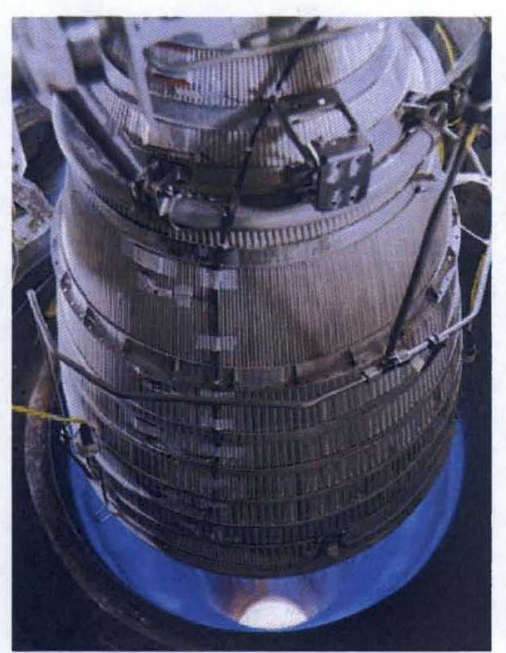

Figure 6. HR 4.01 at $60 \%$ Power

The next two hot-

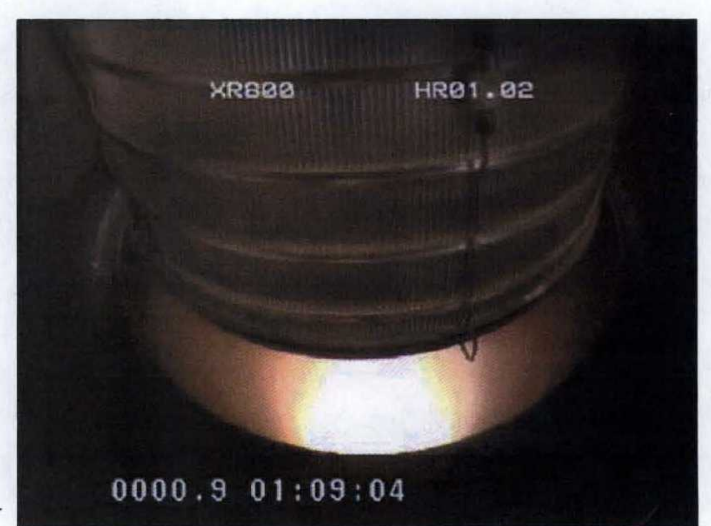

Figure 5: First Ignition of the CECE Engine run attempts occurred on 17 April with run durations of under $2 \mathrm{sec}$, both aborting on a pressure ratio abort that verifies acceleration. The abort programs were adjusted after each run and the subsequent fourth hot-run (HR 4.01) on 18 April successfully completed a full duration run for $23.3 \mathrm{sec}$ at 60 percent power (Figure 6).

HR 5.01 was performed on 30 April for $247.8 \mathrm{sec}$ (Figure 7). CECE successfully demonstrated throttled operation from 80 percent power down to 20 percent power. During the run, the engine achieved the pre-planned power points $(60,80,55,40$, and 30 percent) with no need to adjust mixture ratio from the pre-planned schedules. The test crew then proceeded with the more important objective to further reduce power to the 20 percent level. The Pc level reached target and was held for a few seconds before the test was terminated due to a low fuel system pressure. The advance allowed CECE to demonstrate successful shutdown from 20 percent.

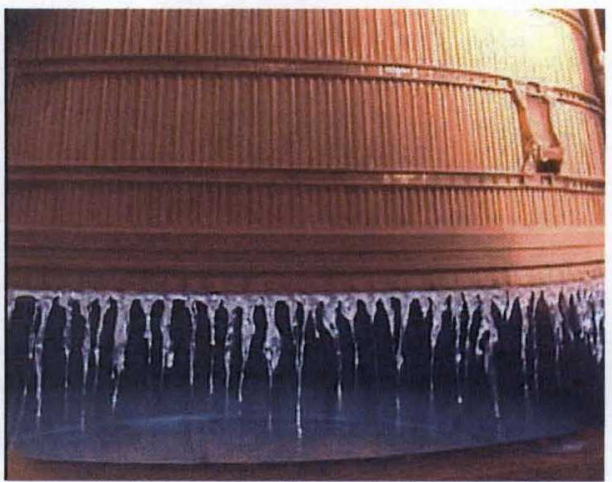

Figure 7. HR 5.01 at 30\% Power 
HR 6.01 was performed on 4 May. The test plan called for a thrust profile run from start to 60 percent power, followed by throttling to achieve $30,20,15,10$ percents, and back up to 40 percent power and shutdown. The engine started and accelerated nominally to 60 percent power, followed by a successful ramp down in power to 30 percent, where all engine parameters remained nominal. However, during the ramp from 30 percent to 20 percent, the engine aborted on a parameter that monitors pump interactions, appearing to be the result of a fuel pump stall. The total duration of the test was $82.5 \mathrm{sec}$. Subsequent data review determined that the fuel pump did experience a stall condition induced by system instability. The valve suite schedule was adjusted from the run's data and the engine deemed ready to proceed.

The next test, HR 7.01, was performed on 5 May, achieving throttle variations ranging from 102 percent power to 30 percent power over a run duration of $241.4 \mathrm{sec}$, including a nominal shutdown. Vibration measurements were low at all times and there were no indications of any issues at shutdown.

HR 8.01 was performed on 6 May and successfully achieved throttled operation to the 10 percent thrust setting (Figure 8) and a little beyond. On the manual approach from 30 percent down to 10 percent, stepwise reductions in Pc were accompanied by stepwise adjustments in other engine control inputs. Once at 10 percent, dwell at power was sustained for approximately $20 \mathrm{sec}$ before test time constraints required proceeding back up to 30 percent power. At 30 percent and stable, all adjustments were zeroed out and the engine proceeded with an automatic ramp up to 80 percent and 90 percent power, with pauses at each point. Assessment of remaining facility steam for the test cell ejector system did not permit further run time, and the test was then advanced to a normal shutdown.

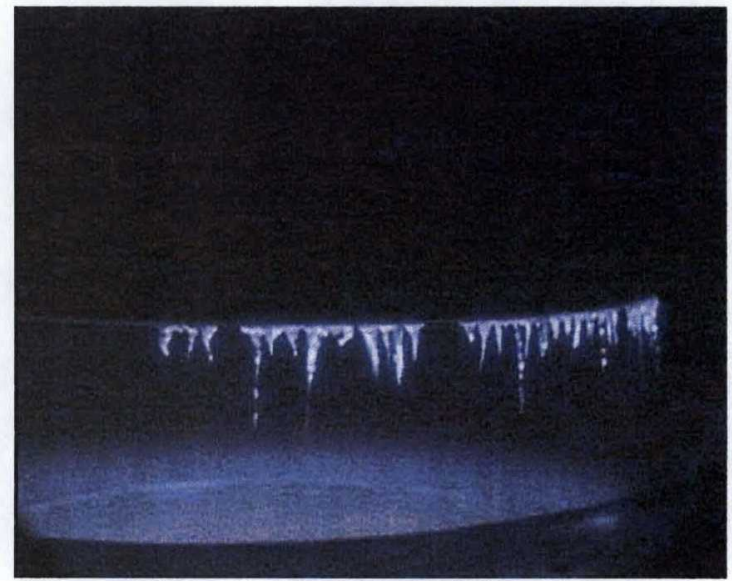

Figure 8. HR 8.01 at 10\% Power

\section{Combustion Stability}

During the last Demo 1 hot-run (HR 8.01), chugging oscillations were encountered at low throttle power levels. A team was formed to understand the significant data observations in the test series with an emphasis on the low power chug instability.

During the investigation of this phenomenon, four separate low frequency stability models, with varying levels of detail, were executed to aid in understanding the phenomenon and to calibrate for predictions to subsequent demonstrator engine tests. Each model had its own idiosyncrasies, assumptions, and level of complexity. However, each model separately has aided in understanding the chugging event during Demo 1 testing. Modeling the CECE low power throttling regime has been a challenge. However, the stability models were successfully calibrated to this regime and provided insight into instability mechanisms.

Although much insight was obtained from the four stability models and calibration to the low power levels was successful, additional testing was deemed desirable to further understand the phenomenon. A second test series was conducted in March and April of 2007 using the same engine configuration. In addition to gathering further engine operability and design data, the test data will be used in part to further define the stability boundaries where the calibrated models can be applied. The analysis of the results of this testing is still in process.

\section{Conclusion}

CECE Demo 1 testing provided critical, early empirical confirmation of detailed component- and engine system-level internal environments and subsystem interactions needed to confirm that 10:1 throttling (with margin) in a LOX/LH2 cryogenic engine is viable for lunar orbit insertion and landing mission segments. The acquired data will serve as a critical database to establish design and analysis confidence for extended throttling to even greater 
levels if necessary for future and evolving Lunar Lander risk reduction development. Successes of the program include:

- A current production cryogenic engine was successfully modified to serve as a deep throttling testbed engine to explore technologies required for ongoing Lunar Lander risk reduction activities.

- Up to 11.4:1 throttling with stable operability was demonstrated in a LOX/LH2 engine in a space-relevant environment.

- Critical deep throttling operability data was acquired at the system and subsystem levels.

- Extensive performance data collected to understand the deep throttling engine operating environment for subsequent design application in Phase II (Demonstrator Engine No. 2) and beyond (Lunar Lander DDT\&E), including critically important detailed data of combustion instability at low power that must be eliminated in subsequent design activity (expected to be performed in Phase II, Option 2 of the CECE program).

Further analysis to better understand the combustion instability oscillations that were experienced in the Demonstrator Engine No. 1 tests at low power were conducted early in the subsequent Phase II, Option 1 program begun in July 2006. The combustion instability must be clearly understood and eliminated in any future design and demonstration activity prior to start of Lunar Lander DDT\&E.

Also conducted under the Phase II, Option 1 program is a follow-on test of the Demo 1 engine (referred to as Demo 1.5) to explore additional engine operating modes of high value, including focused investigation of the chug (combustion instability) phenomena, expanding the envelope of thrust transient operation, and throttled thrust performance impacts associated with mixture ratio excursions. The Demo 1.5 engine test began in late March 2007 and the resulting data will be summarized in a final report.

\section{Acknowledgments}

The authors would like to thank the entire PWR and NASA team for the dedication and effort demonstrated throughout the first twenty-four months of the CECE program.

\section{References}

1. “Advanced RL10 Engine for Manned Lunar Missions", PWR Internal Report PWA FR-388, 18 April 1962.

2. "Discussion of the RL10 Engine Applied to Apollo Propulsion", PWR Internal Report PWA FR-918, 12 March 1964.

3. Colbert, J. E., Mosier, S. A., and Bailey, T. E., "FLOX/Methane Pump-Fed Engine Study Final Report", NASA CR-72485, 10 May 1969.

4. Pugh, Richard L., "The Many Facets of the RL10 Liquid Rocket Engine...A Continuing Success Story", AIAA-98-3680, Technical Paper presented at $34^{\text {th }}$ AIAA/ASME/SAE/ASEE Joint Propulsion Conference, 13-15 July 1998. 